\title{
Identification of key genes and pathways by bioinformatics analysis with TCGA RNA sequencing data in hepatocellular carcinoma
}

\author{
QIANDONG ZHU ${ }^{1,2}$, YUNPENG SUN ${ }^{2}$, QINGQING ZHOU ${ }^{3}$, QIKUAN $\mathrm{HE}^{2}$ and HAIXIN QIAN ${ }^{1}$ \\ ${ }^{1}$ Department of General Surgery, The First Affiliated Hospital of Soochow University, \\ Suzhou, Jiangsu 215006; Departments of ${ }^{2}$ Hepatobiliary Surgery and ${ }^{3}$ Operation Room, \\ The First Affiliated Hospital of Wenzhou Medical University, Wenzhou, Zhejiang 325000, P.R. China
}

Received February 2, 2018; Accepted September 6, 2018

DOI: $10.3892 / \mathrm{mco} .2018 .1728$

\begin{abstract}
Improved insight into the molecular characteristics of hepatocellular carcinoma (HCC) is required to predict prognosis and to develop a new rationale for targeted therapeutic strategy. Bioinformatics methods, including functional enrichment and network analysis combined with survival analysis, are required to process a large volume of data to obtain further information on differentially expressed genes (DEGs). The RNA sequencing data related to HCC in The Cancer Genome Atlas (TCGA) database were analyzed to screen DEGs, which were separately submitted to perform gene enrichment analysis to identify gene sets and signaling pathways, and to construct a protein-protein interaction (PPI) network. Subsequently, hub genes were selected by the core level in the network, and the top hub genes were focused on gene expression analysis and survival analysis. A total of 610 DEGs were identified, including 444 upregulated and 166 downregulated genes. The upregulated DEGs were significantly enriched in the Gene Ontology analysis (GO): Cell division and in the Kyoto Encyclopedia of Genes and Genomes (KEGG) pathway: Cell cycle, whereas the downregulated DEGs were enriched in GO: Negative regulation of growth and in the KEGG pathway: Retinol metabolism, with significant differences. Cyclin-dependent kinase (CDK)1 was selected as the top hub gene by the PPI network, which
\end{abstract}

Correspondence to: Dr Haixin Qian, Department of General Surgery, The First Affiliated Hospital of Soochow University, 188 Shizi Street, Suzhou, Jiangsu 215006, P.R. China

E-mail: uplimit@163.com

Abbreviations: HCC, hepatocellular carcinoma; DEGs, differentially expressed genes; TCGA, The Cancer Genome Atlas; GO, Gene Ontology; KEGG, Kyoto Encyclopedia of Genes and Genomes; GEO, Gene Expression Omnibus; FC, fold change; FDR, false discovery rate; PPI, protein-protein interaction; OS, overall survival; CDKs, cyclin-dependent kinases

Key words: hepatocellular carcinoma, prognosis, The Cancer Genome Atlas, bioinformatics exhibited a similar expression trend with the data from the Gene Expression Omnibus (GEO) database. Survival analysis revealed a significantly negative correlation between CDK1 expression level and overall survival in the TCGA group $(\mathrm{P}<0.01)$ and the GEO group $(\mathrm{P}<0.01)$. Therefore, high-throughput TCGA data analysis appears to be an effective method for screening tumor molecular markers, and high expression of CDK1 is a prognostic factor for HCC.

\section{Introduction}

Hepatocellular carcinoma (HCC) is one of the most common types of cancer $(1,2)$, with $>780,000$ new cases and $\sim 745,000$ deaths annually worldwide (3). Unfortunately, there are only a few treatment options, with unsatisfactory efficacy. Tumor stage is a decisive factor in the selection of treatment strategy. Liver transplantation and hepatectomy are the best potentially curative treatment regimens for patients with HCC. However, a number of patients with advanced-stage $\mathrm{HCC}$ are not eligible for these treatments. The 5-year survival rate for advanced HCC currently remains poor. Despite a number of studies attempting to elucidate the mechanism underlying tumorigenesis, no method has been found to be suitable for the entire patient population due to the lack of specificity and sensitivity. Therefore, it is urgent to identify sensitive and specific biomarkers for HCC progression and to elucidate the molecular mechanisms involved in HCC progression to predict prognosis and to develop a novel targeted therapeutic strategy.

Genome-wide expression profiles have recently been used to identify differentially expressed genes (DEGs) during disease progression $(4,5)$, which enables the identification of candidate biomarkers for the diagnosis, therapy and prognosis of tumors. The high-throughput platforms of gene expression are the base of genome-wide regulatory and interaction networks $(5,6)$. Recently, next-generation sequencing accompanied by higher throughput developed rapidly, allowing more accurate and comprehensive examination of global gene expression profiles (7). However, to date, there have only been a few studies with a small sample size that used RNA sequencing data in the transcriptomic landscape of $\operatorname{HCC}(8,9)$, 
and it is imperative to further elucidate the prominent role of whole-transcriptome sequencing in HCC.

The Cancer Genome Atlas (TCGA) is a project that was initiated in 2005 by the National Cancer Institute to identify genetic mutations implicated in cancer using genome sequencing and bioinformatics. A large number of tissue samples are stored in the TCGA database and examined from multiple aspects, including genomic expression. In addition, the data are freely available to all researchers for their individual studies (7). Therefore, the large TCGA RNA sequencing (RNAseq) dataset was used in the present study.

All RNAseq data and clinical data were extracted from the TCGA database of HCC. Global gene expression changes were compared between tumor tissues (T) and adjacent non-tumor tissues (NT) and numerous DEGs were identified. Then, DEGs were subjected to a gene enrichment analysis with an online functional annotation tool to identify gene sets and signaling pathways that were significantly enriched with DEGs, and to construct a protein-protein interaction (PPI) network and obtain hub genes for survival analysis. Then, the top hub gene, cyclin-dependent kinase (CDK)1, which is crucial for the mitotic process, was further analyzed and validated with Gene Expression Omnibus (GEO) data to conduct a survival analysis, in order to determine whether CDK1 expression is directly associated with survival time and prognosis in $\mathrm{HCC}$.

\section{Materials and methods}

TCGA data of HCC. All available TCGA data on HCC were obtained from the TCGA data portal (TCGA group). In September 2016, there were RNAseq data on 424 HCC samples, including 324 single tumor samples, 50 pairs of $\mathrm{HCC}$ and adjacent non-tumor liver tissues, and clinical data including survival time and survival status records of 370 patients (excluding 1 case without survival time and 3 cases of recurrence). The data, which had been generated using the Illumina HiSeq 2000 platform, were annotated to a reference transcript set of Human GRCh38/hg38 gene standard track.

GEO data of HCC. We extracted a microarray expression profile (GEO group) from the GEO database. All 247 patients with HCC included in the GSE14520 profile were identified; 26 patients were excluded from this study, including 22 patients on GPL571 and 5 without outcome data (1 case was on GPL571 and had no outcome data). Finally, 221 patients carried out on GPL3921 were included in the present analysis. All liver tissues were obtained from patients who underwent radical resection between 2002 and 2003 at the Liver Cancer Institute and Zhongshan Hospital Affiliated to Fudan University. Tumor sample processing and microarray analysis were performed as reported by Roessler et al $(10,11)$.

Global gene expression analysis. Differential gene expression analysis with RNAseq data was performed using $\mathrm{R}$ package edgeR $(7,12)$. As suggested by edgeR, genes of low read counts are usually not of interest in DGE analysis. Therefore, an average raw read count for each gene $>1$ was applied to determine candidate genes that were reasonably expressed. The T/NT expression fold change (FC) denotes

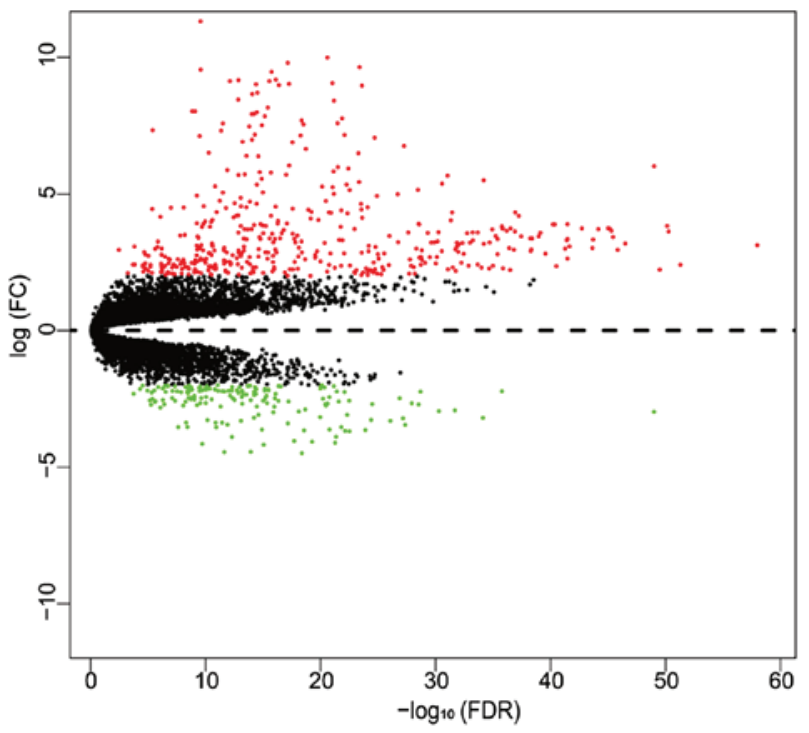

Figure 1. Volcano plot of the differentially expressed genes (DEGs) with low expression genes $(\log \mathrm{CPM} \leq 1)$ excluded between $\mathrm{T}$ and NT. Y-axis: $\log \mathrm{FC}$ (fold change); X-axis: $-\log _{10}$ (FDR) for each gene; the color of the data points denotes the status of DEGs (red points: FC $>2$ with FDR $<0.05$; green points: FC $<2$ with FDR $<0.05)$. CPM, counts per million; FDR, false discovery rate; $\mathrm{T}$, tumor tissues; NT, non-tumor tissues.

upregulation or downregulation according to the FC value. Subsequently, $\log \mathrm{FC}, \log \mathrm{CPM}, \mathrm{P}$-value and the corresponding false discovery rate (FDR) were all reported by the $\mathrm{R}$ package. FDR $<0.05, \log \mathrm{CPM}>1$ and $\log \mathrm{FCl}>2$ were set as inclusion criteria for DEG selection. The gene expression level based on microarray data was calculated using $\mathrm{R}$ package limma with RMA correction.

Gene ontology (GO) and pathway enrichment analysis. GO analysis is a useful method for annotating genes and gene sets with biological characteristics for high-throughput genome or transcriptome data (13). The Kyoto Encyclopedia of Genes and Genomes (KEGG) pathway is a knowledge base for systematic analysis of gene functions (14). The Database for Annotation, Visualization and Integrated Discovery (DAVID) provides a comprehensive set of efficient and concise annotation tools for researchers to understand the biological meaning behind numerous genes (15). GO and KEGG pathway enrichment analysis were used for DEGs using the DAVID database. FDR $<0.05$ was set as the cut-off criterion for the two analyses.

PPI network. PPI networks can provide information on the molecular mechanism underlying cellular activity. In the present study, a PPI network of DEGs was constructed using an online database, the Search Tool for the Retrieval of Interacting Genes (STRING), which is designed for evaluating PPI information. STRING (version 9.0) covers 1,133 organisms, including 5,214,234 proteins (16). We mapped DEGs to STRING to identify the interactive relationships among DEGs. A confidence score of 0.4 was set as the cut-off criterion, and the top 10 DEGs of node degrees were selected as hub genes.

Statistical analysis. All data analyses were performed in the $\mathrm{R}$ programming environment (version 3.2.5) and 


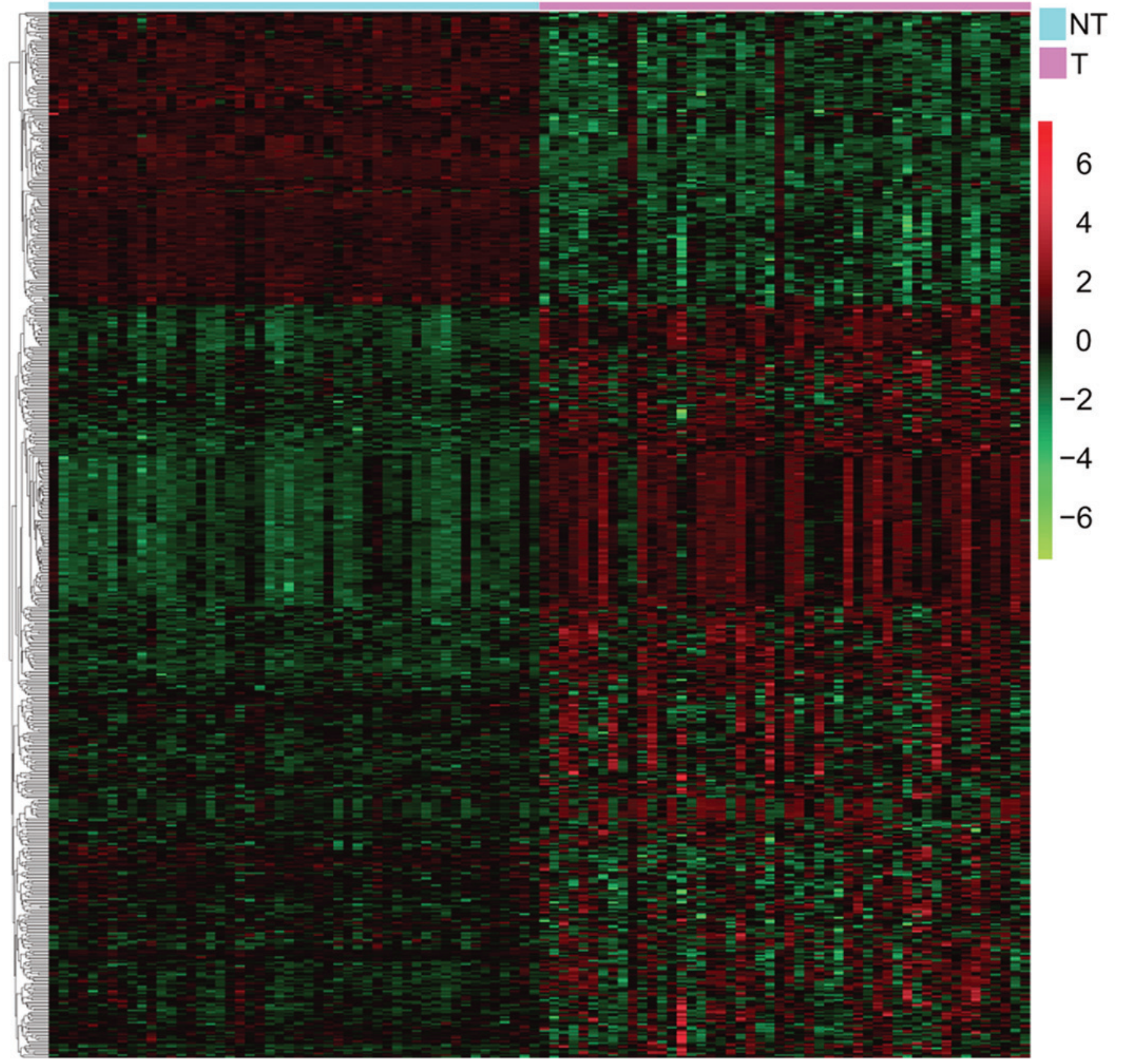

Figure 2. Heatmap of differentially expressed genes (444 upregulated and 166 downregulated genes). Red, upregulation; green, downregulation. FDR $<0.05$, $\log \mathrm{CPM}>1$ and $\log \mathrm{FCl}>2$ were set as the cut-off criterion. FC, fold change; CPM, counts per million; FDR, false discovery rate; T, tumor tissues; NT, non-tumor tissues.

Bioconductor (17). Statistical analyses were carried out with GraphPad Prism 5.0 software (GraphPad Software, Inc., La Jolla, CA, USA). Single comparisons between two groups were performed with the Student's t-test. Survival analysis was performed according to the Kaplan-Meier analysis and log-rank test. Overall survival (OS) was defined as the time between the date of surgery and date of death or the date of the last follow-up. P-values $<0.05$ were considered to indicate statistically significant differences.

\section{Results}

Identification of DEGs. In the present study, gene expression profiles from TCGA were utilized to compare gene expression between T and NT. By comparing the RNAseq read counts of the various genes and subsequently applying the cut-off criteria, 610 genes were identified as DEGs, including 444 upregulated and 166 downregulated genes. High expression genes $(\log C P M>1)$ were included in the volcano plot, with low expression genes excluded (Fig. 1). Subsequently, a heatmap of DEGs was created; the mRNA expression profiles of T and NT resulted in obviously separate clusters (Fig. 2).

Gene set enrichment analysis. To gain further insight into the function of identified DEGs for HCC, gene enrichment analysis was performed using DAVID, including GO and KEGG pathway enrichment analyses.Enrichment analyses of the upregulated and downregulated genes were performed separately, as previously 

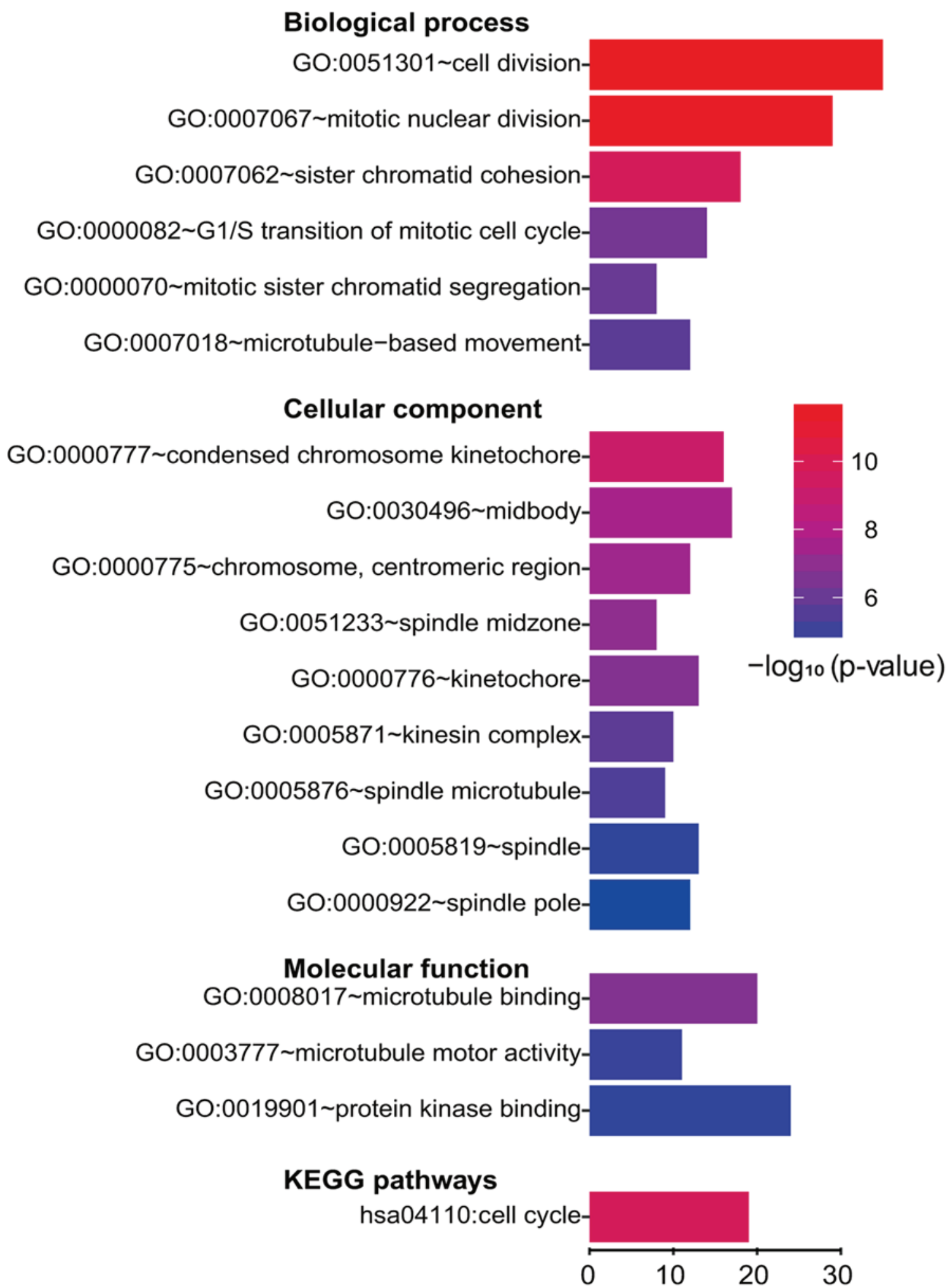

Figure 3. Gene ontology (GO) and Kyoto Encyclopedia of Genes and Genomes (KEGG) pathway enrichment analysis of upregulated differentially expressed genes. Count: Number of genes related to the enriched GO or KEGG pathway. The color of the bar denotes $-\log _{10}(\mathrm{P}-\mathrm{value})$.

recommended (18). By subjecting the upregulated genes to enrichment analysis, we observed numerous enriched gene sets. For GO biological process, the genes were mainly enriched in cell division. For GO cellular component, the gene enrichment mainly involved condensed chromosome and spindle. For GO molecular function, microtubule binding and protein kinase were implicated (Fig. 3). A number of downregulated genes were significantly enriched in various GO domains (Fig. 4). The biological process was associated with negative regulation of growth and mineral ion response, cellular component involved extracellular region and organelle membrane, and molecular function was related to heme binding and enzyme activity. 


\section{Biological process}

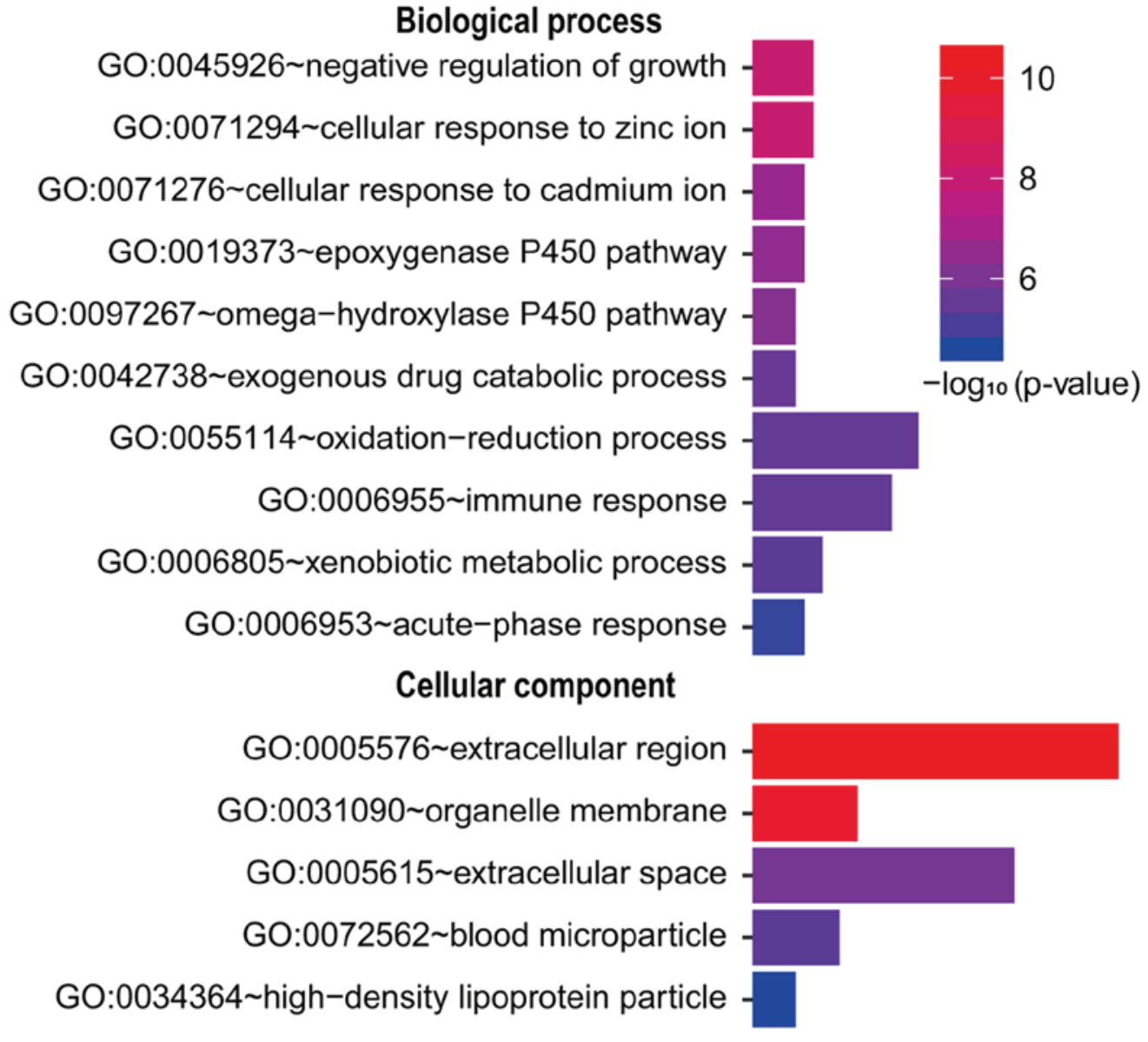

Molecular function

GO:0020037 heme binding

GO:0019825 oxygen binding

GO:0016705 oxidoreductase activity

GO:0004497 monooxygenase activity

GO:0005506 iron ion binding

GO:0008392 arachidonic acid epoxygenase activity

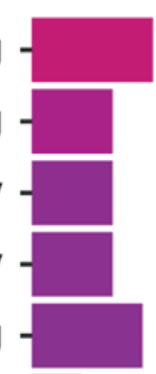

KEGG pathways

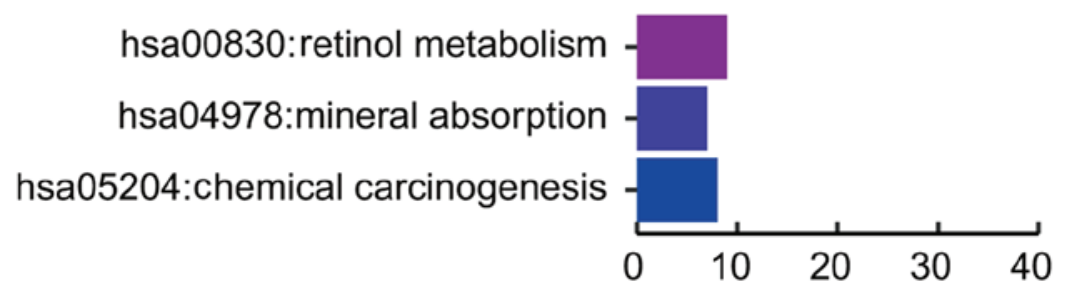

Figure 4. Gene ontology (GO) and Kyoto Encyclopedia of Genes and Genomes (KEGG) pathway enrichment analysis of downregulated differentially expressed genes. Count: Number of genes related to the enriched GO or KEGG pathway. The color of the bar denotes $-\log _{10}(\mathrm{P}-\mathrm{value})$.

We further investigated the functional implications of these DEGs in the development of HCC by KEGG pathway analysis. A number of DEGs were enriched in four KEGG pathways, including Cell cycle in upregulated DEGs and Retinol metabolism, Mineral absorption and Chemical carcinogenesis in downregulated genes (Figs. 3 and 4).
PPI network. The PPI network of DEGs was constructed, which consisted of 568 nodes and 1,952 edges, with a mean node degree of 6.87 . The top 10 genes were selected as hub genes by degree, such as CDK1, TOP2A, CCNB1, CDC20, PLK1, BIRC5, CCNB2, FOS, AURKA and AURKB (Fig. 5). Subsequently, the hub genes were again submitted to STRING 


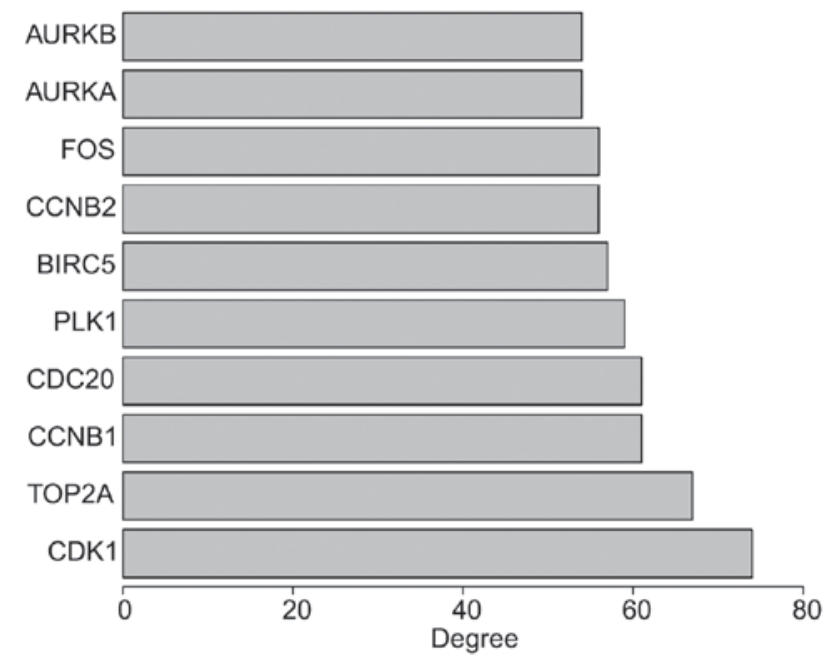

Figure 5. The hub genes selected by degrees from the protein-protein interaction network of differentially expressed genes. Degree stands for the core level of genes.

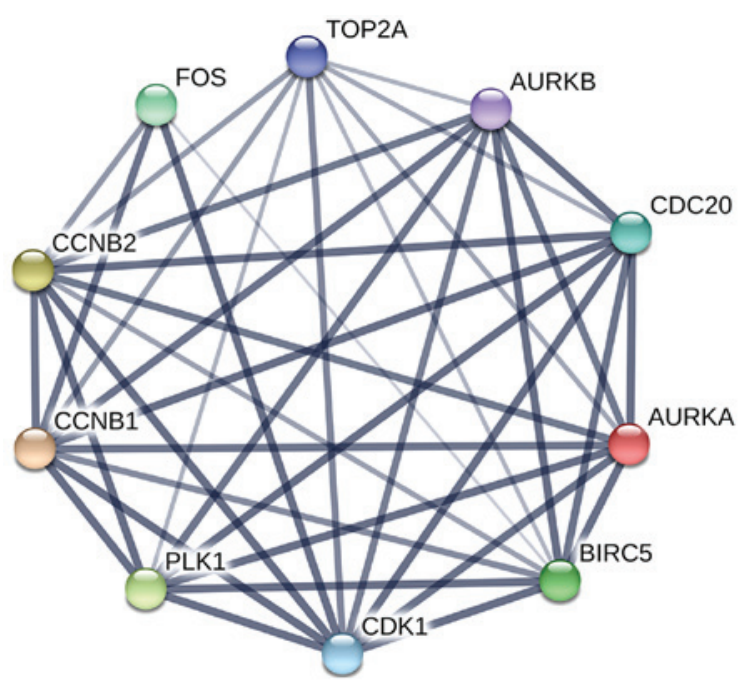

Figure 6. Protein-protein interaction network of the hub genes. The lines represent interaction associations between nodes and line thickness indicates the strength of data support.

to verify the interaction among them. The PPI network consisted of 10 nodes and 40 edges, with a mean node degree of 8 , and showed a closer protein interaction among the hub genes (Fig. 6). Then, the top hub gene, CDK1, was selected as a candidate gene for further analysis.

CDK1 overexpression and correlation with hub genes. Next, to validate dysregulated expression of CDK1, we analyzed its expression data separately with 50 pairs of $\mathrm{T}$ and NT, and all samples in the TCGA group. These data confirmed that CDK1 was significantly overexpressed in HCC compared with adjacent NT tissues (Fig. 7A and B), with $>2$-fold increase in CDK1 expression in 98\% (49/50) of the tumors (Fig. 8). A similar result was found in the GEO group (Fig. 7C). To further investigate the link between CDK1 and the other hub genes, Pearson's correlation was used and revealed a statistically significant correlation between CDK1 and TOP2A $(\mathrm{R}=0.96$,
$\mathrm{P}<0.01), \mathrm{CCNB} 1(\mathrm{R}=0.91, \mathrm{P}<0.01), \mathrm{CDC} 20(\mathrm{R}=0.90, \mathrm{P}<0.01)$, PLK1 $(\mathrm{R}=0.91, \mathrm{P}<0.01), \mathrm{BIRC5}(\mathrm{R}=0.93, \mathrm{P}<0.01), \mathrm{CCNB} 2$ $(\mathrm{R}=0.94, \mathrm{P}<0.01)$, FOS $(\mathrm{R}=-0.63, \mathrm{P}<0.01)$, AURKA $(\mathrm{R}=0.81$, $\mathrm{P}<0.01)$ and AURKB $(\mathrm{R}=0.90, \mathrm{P}<0.01)$ (Fig. 9).

Survival analysis. In the TCGA group, a total of 370 cases of HCC patients were enrolled in the study. The patients were divided into two groups according to gene expression, and expression levels higher than the median were classified into the high expression group; otherwise, they were classified into the low expression group. OS was calculated based on gene expression. Subsequently, survival analysis was performed to determine the association between the gene expression level and patient OS. We found that the expression level of the hub genes, except FOS, was negatively correlated with OS, with a statistically significant difference $(\mathrm{P}<0.01$; Fig. 10A). We further analyzed the data to validate our findings in the GEO group. Similarly, Kaplan-Meier and log-rank test analysis revealed that the gene expression level was negatively correlated with OS (Fig. 10B). To summarize, high expression of CDK1 was shown to predict a worse prognosis in patients with HCC.

\section{Discussion}

Despite the surgical and medical advances in the treatment of HCC patients, the overall mortality has remained unchanged over the past decades (2) and the molecular mechanism underlying the development of this cancer has not been fully elucidated. HCC remains one of the most common causes of cancer-related morbidity and mortality. HCC is very difficult to detect at an early stage, and there are currently no effective treatments for patients with advanced-stage disease. Therefore, it is crucial to improve survival rate and prognosis through understanding the etiological factors and molecular mechanisms involved in HCC. Recently, microarray technology has rapidly developed and has been widely applied to identify general genetic alterations in malignant diseases, such as HCC $(19,20)$. With the recent technological advances, next-generation sequencing enables a more comprehensive and accurate examination of global gene expression profiles. High-throughput analyses are used to identify gene expression signatures to improve the accuracy of prognosis (21).

In order to identify potential biomarkers for HCC prognosis and therapy, we used data from TCGA to access valuable information on liver cancer. A total of 610 DEGs were identified, including 444 upregulated and 166 downregulated genes. To further elucidate the underlying function of DEGs, functional enrichment analysis based on GO and pathway enrichment analysis based on KEGG were performed using DAVID. These upregulated genes were mainly enriched in the GO and pathways related to proliferation, such as cell division and cell cycle, while the downregulated genes were mainly enriched in negative regulation of growth, immune response, redox reactions and signal transmission. Numerous abnormally modified GO and KEGG pathways were closely associated with cancer. Redox reactions and biological metabolism are important for maintaining normal life activities. Recent studies have demonstrated that normal immune function is crucial for the prevention and treatment 

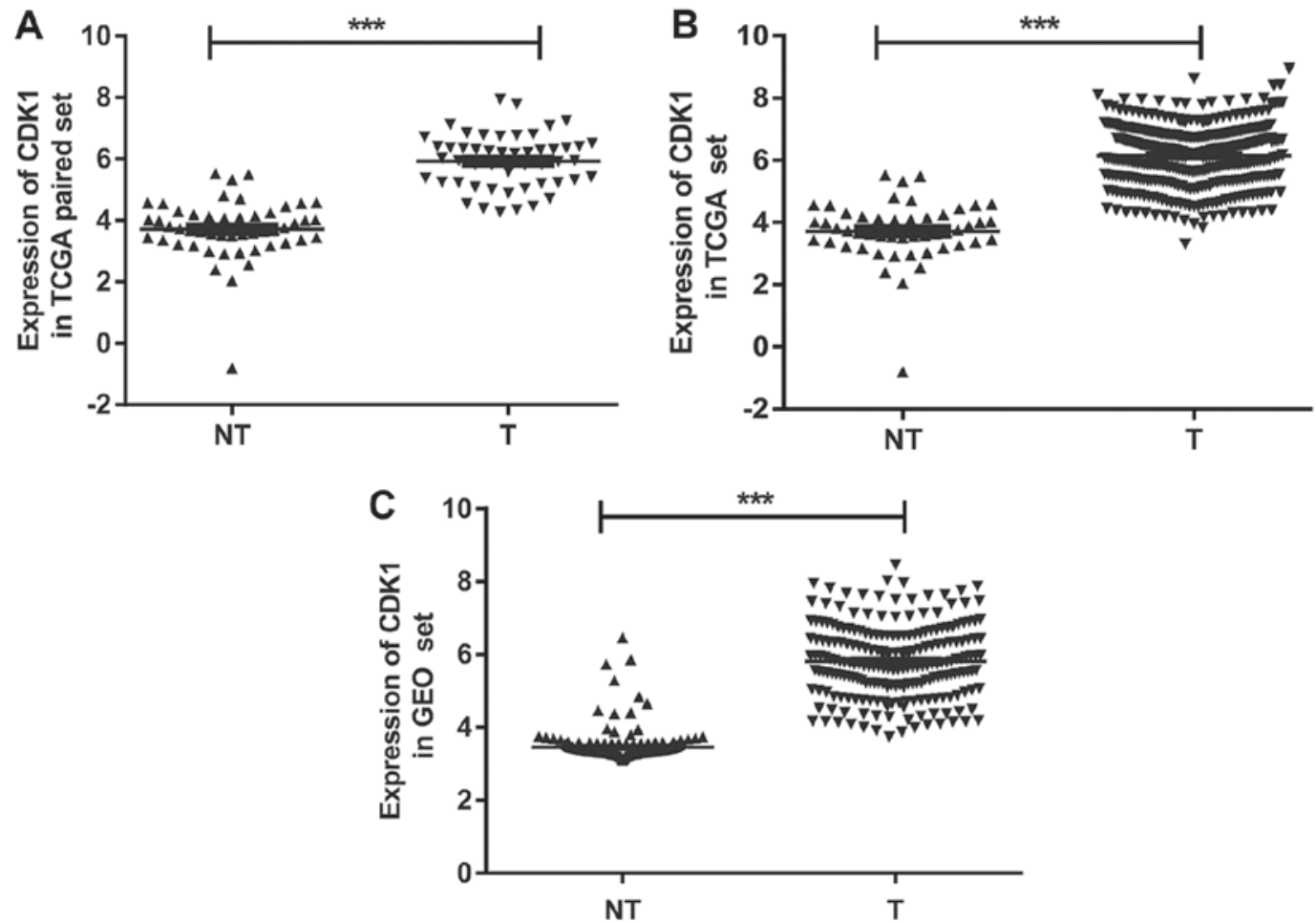

Figure 7. CDK1 significantly overexpressed in tumor tissue (T) compared with adjacent non-tumor liver tissues (NT) in the (A) TCGA paired set (50 pairs of samples from TCGA); (B) TCGA set (all samples from TCGA); and (C) GEO set (samples from GEO). ${ }^{* * *} \mathrm{P}<0.01$. CDK, cyclin-dependent kinase; TCGA, The Cancer Genome Atlas; GEO, Gene Expression Omnibus; T, tumor tissues; NT, non-tumor tissues.

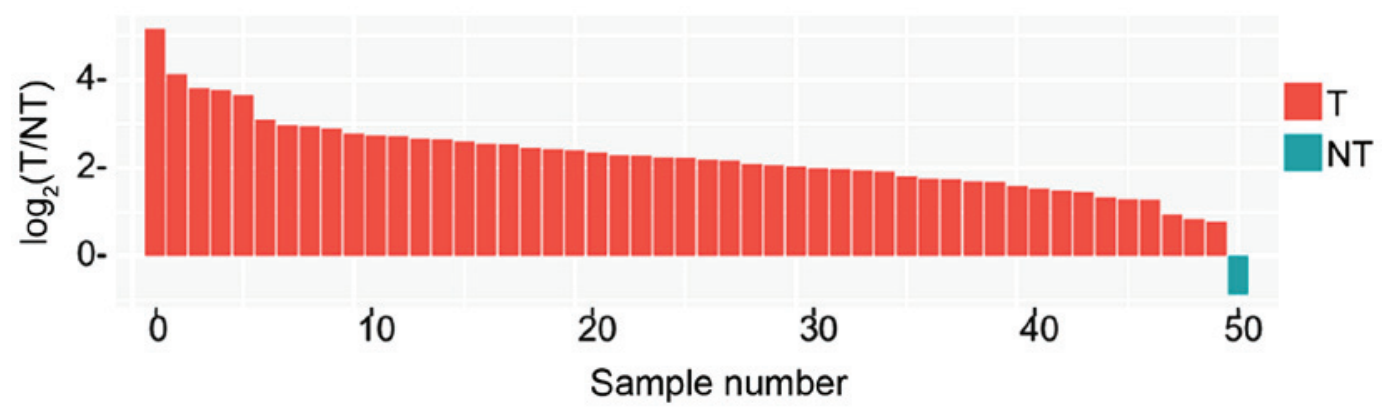

Figure 8. Waterfall plot demonstrating the distribution of CDK1 expression in TCGA data. These data confirmed that CDK1 was significantly overexpressed in HCC when compared with surrounding normal tissues, with a $>2$-fold increase in CDK1 expression in 98\% (49/50) of the tumors. CDK, cyclin-dependent kinase; TCGA, The Cancer Genome Atlas; HCC, hepatocellular carcinoma T, tumor tissues; NT, non-tumor tissues.

of tumors $(22,23)$. Reprogramming energy metabolism and evading immune destruction are considered to be two newly emerging hallmarks, which are as important as uncontrolled proliferation and evasion of apoptosis in tumorigenesis and tumor progression $(24,25)$. Our results demonstrated a liver cancer cell state of enhanced proliferation and division, reduced negative growth regulation, redox electron transport and immune function.

Among these DEGs, a closely interacting PPI network was found, including 10 genes, namely CDK1, TOP2A, CCNB1, CDC20, PLK1, BIRC5, CCNB2, FOS, AURKA and AURKB, which were again analyzed by DAVID and were found to be associated with cell division and proliferation. CDK1 had a highest degree in the PPI network and was considered as the top hub gene. Pearson's correlation analysis revealed that the expression of other hub genes exhibited a significantly positive correlation with CDK1, except FOS, the expression of which had a significantly negative correlation with tumors.

The cell cycle is the series of events that occur during cell division and DNA duplication, and it is an evolutionarily conserved process necessary for mammalian cell growth and development. For cells to accurately duplicate their contents and divide, they must proceed through the steps of the cell cycle in a specific order. Loss of normal cell cycle control is a hallmark of human cancer (26). Tumor cells accumulate genetic alterations that lead to unscheduled cell proliferation and genomic instability. Chromosomal instability is correlated with poor prognosis in multiple solid tumors, indicating that increasing genetic diversity contributes to altered tumor cell survival and chemoresistance (27). At present, several cell cycle-related genes have been reported to be involved in HCC initiation and progression $(28,29)$. Our results 

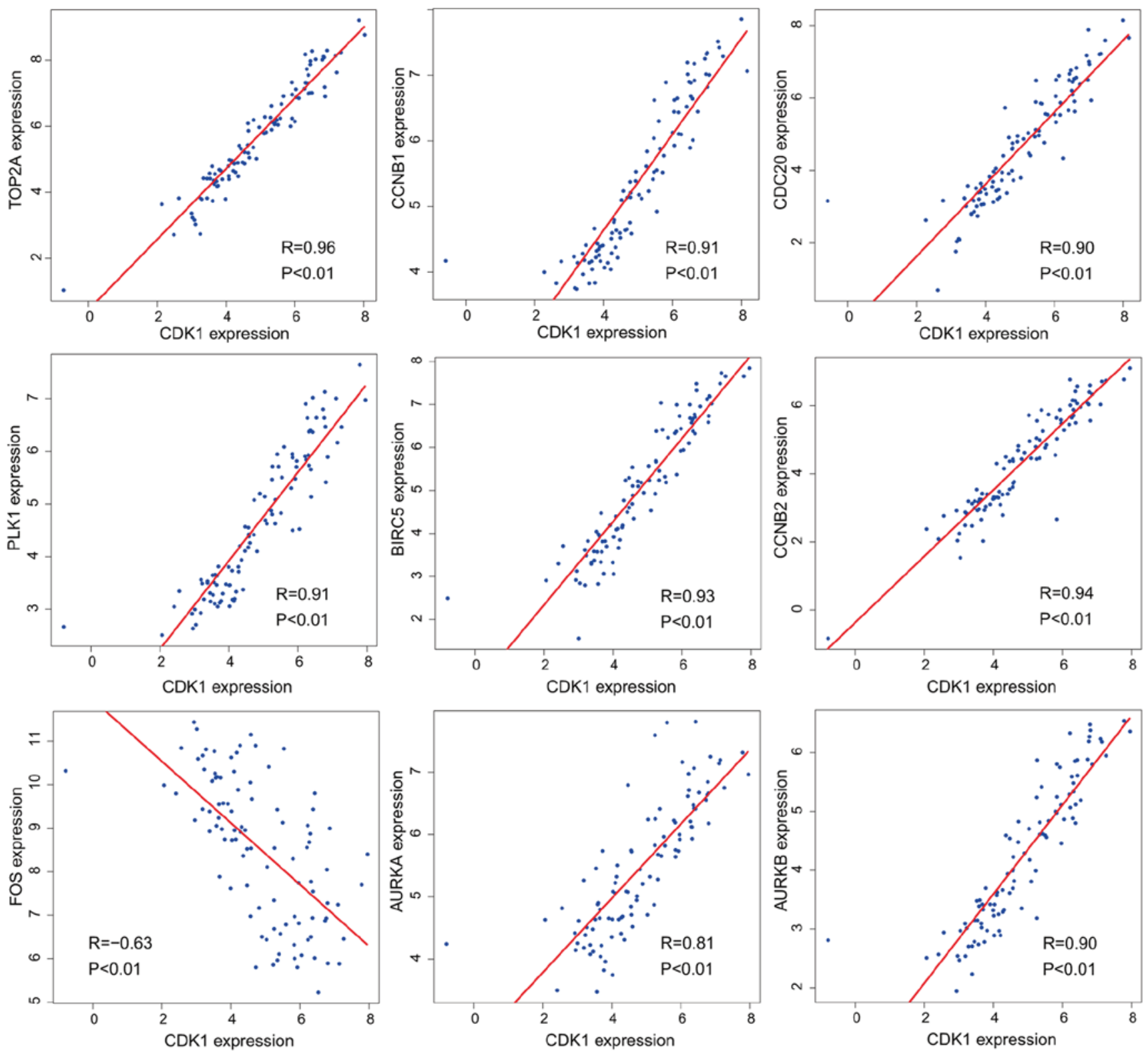

Figure 9. Pearson's correlation analysis confirmed that there was a statistically significant correlation between $\mathrm{CDK} 1$ and the other hub genes: TOP2A ( $\mathrm{R}=0.96$, $\mathrm{P}<0.01)$, CCNB1 ( $\mathrm{R}=0.91, \mathrm{P}<0.01), \mathrm{CDC} 20(\mathrm{R}=0.90, \mathrm{P}<0.01)$, PLK1 $(\mathrm{R}=0.91, \mathrm{P}<0.01), \mathrm{BIRC}$ ( $\mathrm{R}=0.93, \mathrm{P}<0.01), \mathrm{CCNB} 2(\mathrm{R}=0.94, \mathrm{P}<0.01), \mathrm{FOS}(\mathrm{R}=-0.63$, $\mathrm{P}<0.01)$, AURKA $(\mathrm{R}=0.81, \mathrm{P}<0.01)$ and AURKB $(\mathrm{R}=0.90, \mathrm{P}<0.01)$. CDK, cyclin-dependent kinase.
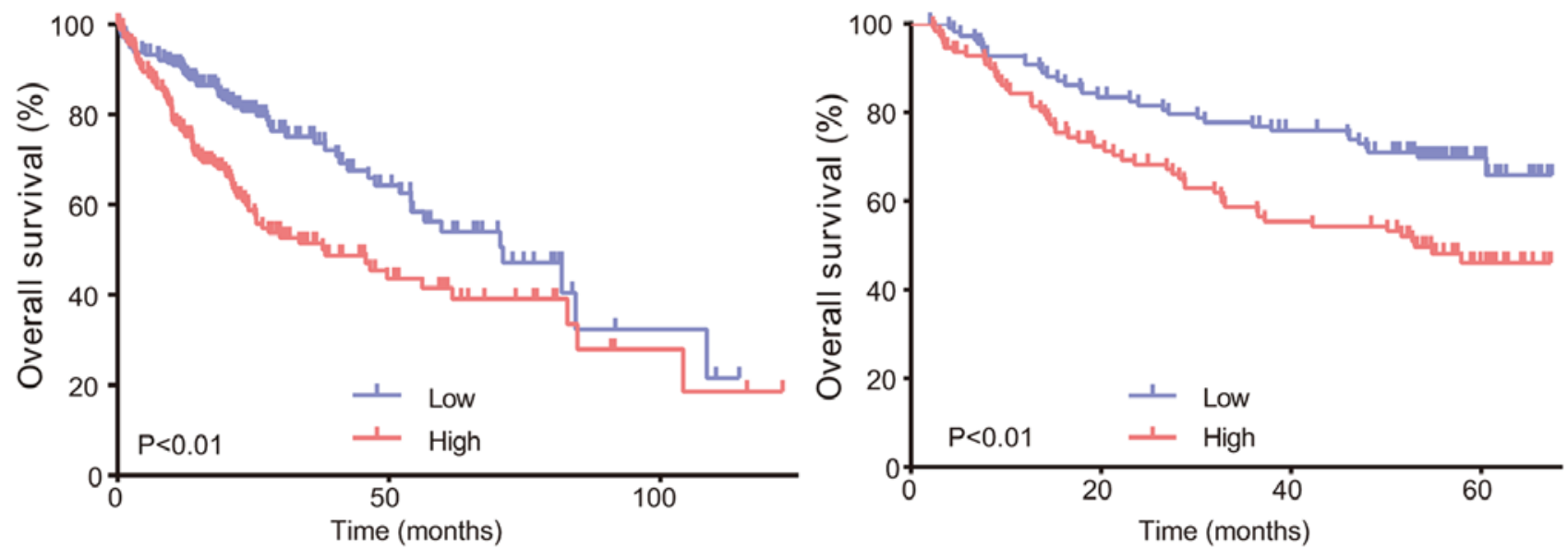

Figure 10. The expression level of CDK1 was negatively associated with overall survival with a significant difference in TCGA (left panel, P<0.01) and GEO (right panel, $\mathrm{P}<0.01$ ). CDK, cyclin-dependent kinase; TCGA, The Cancer Genome Atlas; GEO, Gene Expression Omnibus. 
demonstrated that a large number of DEGs were significantly enriched in the molecular process related to cell cycle, cell division and growth regulation, demonstrating that cell cycle and cell regulation disorders are crucial for tumorigenesis in HCC.

CDKs are master regulatory kinases and coordinate all cell cycle events. During the entire cell cycle, cyclins, which are expressed periodically, and CDKs, which are relatively stable, combine to form a complex, which activates the kinase activity of CDKs and can precisely regulate the cell cycle at different phases through inducing or inhibiting the expression of several genes necessary for cells to enter mitosis. CDK1 is a catalytic subunit of $\mathrm{M}$ phase-promoting factor, which acts mainly towards the end of the G2 phase and can direct cells into the mitotic $\mathrm{M}$ phase. Cyclin B synthesis begins in the late $\mathrm{G} 1$ phase, increases in the $\mathrm{S}$ phase, peaks in the late $\mathrm{G} 2$ and $\mathrm{M}$ phase, then enters the nucleus and binds to CDK1. When the cells exit the $\mathrm{M}$ phase, cyclin $\mathrm{B}$ is degraded, the kinase activity of CDK1 is inactivated, and the cells are guided into the next cell cycle (30). CCNB1 overexpression promotes cell proliferation and tumor growth in human colorectal cancer (31), and is a poor prognostic factor for breast cancer (32).

PLK1 plays an extremely important role in the replication of hepatitis B virus, tumor metastasis and autophagy (33-35).

TOP2A, as a common predictor of chemotherapy efficacy, exhibits a significant correlation between its amplification or deletion and the reactivity to anthracyclines (36). Patients with high expression of TOP2A were found to be more sensitive to anthracyclines, while patients with low expression were resistant to these agents (37).

FOS, an AP-1 transcription factor subunit, is involved in mediating a number of biological processes, such as cell proliferation, differentiation and death (38).

The aurora kinases (AURK) are an evolutionarily conserved family of serine/threonine kinases related to mitosis and meiosis, and most mitotic cells express two AURK isoforms (AURKA and AURKB). These kinases, as molecular switches, regulate multiple processes in cell division, including spindle organization, chromosome alignment, the spindle assembly checkpoint and cytokinesis, among others (39).

CDC20 is an important cofactor of the anaphase-promoting complex or cyclosome (APC/C) E3 ubiquitin ligase by regulating $\mathrm{APC} / \mathrm{C}$ ubiquitin activity on specific substrates for their subsequent degradation by the proteasome. It plays an important role in chromosome segregation and mitotic exit as a target of the spindle assembly checkpoint (40).

BIRC5, a member of the inhibitor of apoptosis protein (IAP) family, plays an important role in apoptosis, proliferation and angiogenesis, and is an important prognostic marker and survival factor $(41,42)$.

In the present study, the expression of the hub genes, except FOS, was found to be significantly increased in HCC, and the expression level was negatively correlated with OS, with a statistically significant difference. These results were similar to the expression in other solid tumors (4,43-45), showing the characteristics of the hub genes as oncogenes and the key role of the PPI network in tumorigenesis and tumor progression.

Taken together, the results of the present study demonstrated that several pathways are altered and numerous hub genes, including CDK1, are overexpressed in $\mathrm{HCC}$, with the expression level being significantly associated with survival time; they may be indicative of poor prognosis and may be valuable as prognostic markers for HCC patients. It may be preferable to study these genes as a whole in the context of a PPI network for further analysis, as this will hopefully provide new insights into the molecular mechanisms, prevention and treatment of HCC.

There were certain limitations to the present study, as the results from the RNASeq and bioinformatics lack corresponding experiments in vitro and in vivo. Functional research is necessary to uncover the molecular mechanisms interlinking DEGs in HCC and their role in prognosis and therapy.

\section{Competing interests}

The authors declare that they have no competing interests.

\section{Acknowledgements}

Not applicable.

\section{Funding}

This study was supported in part by the Wenzhou Science and Technology Project (grant no. Y20170096), the Zhejiang Provincial Natural Science Foundation of China (grant no. LY18H160056) and the Science Technology Department of Zhejiang Province Project (grant no. 2016C37127). Fund body only provided a part of the financial support for our study, but did not participate in or interfere with our research design, data collection, data interpretation, or manuscript writing.

\section{Authors' contributions}

QiaZ wrote the manuscript and interpreted the data. HQ designed the study as director. YS performed data mining. QinZ helped correct the manuscript. QH performed data analysis and statistical analysis. All the authors have read and approved the final version of this manuscript.

\section{Ethics approval and consent to participate}

Not applicable.

\section{Patient consent for publication}

Not applicable.

\section{Availability of data and materials}

The datasets generated and analysed in the present study are available from the corresponding author on reasonable request.

\section{References}

1. El-Serag HB: Hepatocellular carcinoma. N Engl J Med 365: 1118-1127, 2011.

2. Villanueva A and Llovet JM: Liver cancer in 2013: Mutational landscape of HCC-the end of the beginning. Nat Rev Clin Oncol 11: 73-74, 2014. 
3. Ferlay J, Soerjomataram I, Dikshit R, Eser S, Mathers C, Rebelo M, Parkin DM, Forman D and Bray F: Cancer incidence and mortality worldwide: Sources, methods and major patterns in GLOBOCAN. Int J Cancer 136: E359-E386, 2015.

4. Shi YX, Zhu T, Zou T, Zhuo W, Chen YX, Huang MS, Zheng W, Wang CJ, Li X, Mao XY, et al: Prognostic and predictive values of CDK1 and MAD2L1 in lung adenocarcinoma. Oncotarget 7: 85235-85243, 2016.

5. Liang B, Li C and Zhao J: Identification of key pathways and genes in colorectal cancer using bioinformatics analysis. Med Oncol 33: 111, 2016.

6. Lee JS and Thorgeirsson SS: Comparative and integrative functional genomics of HCC. Oncogene 25: 3801-3809, 2006.

7. Ho DW, Kai AK and Ng IO: TCGA whole-transcriptome sequencing data reveals significantly dysregulated genes and signaling pathways in hepatocellular carcinoma. Front Med 9 322-330, 2015.

8. Huang Q, Lin B, Liu H, Ma X, Mo F, Yu W, Li L, Li H, Tian T, Wu D, et al: RNA-Seq analyses generate comprehensive transcriptomic landscape and reveal complex transcript patterns in hepatocellular carcinoma. PLoS One 6: e26168, 2011.

9. Lin KT, Shann YJ, Chau GY, Hsu CN and Huang CY: Identification of latent biomarkers in hepatocellular carcinoma by ultra-deep whole-transcriptome sequencing. Oncogene 33: 4786-4794, 2014.

10. Roessler S, Jia HL, Budhu A, Forgues M, Ye QH, Lee JS, Thorgeirsson SS, Sun Z, Tang ZY, Qin LX and Wang XW: A unique metastasis gene signature enables prediction of tumor relapse in early-stage hepatocellular carcinoma patients. Cancer Res 70: 10202-10212, 2010.

11. Roessler S, Long EL, Budhu A, Chen Y, Zhao X, Ji J, Walker R, Jia HL, Ye QH, Qin LX, et al: Integrative genomic identification of genes on $8 \mathrm{p}$ associated with hepatocellular carcinoma progression and patient survival. Gastroenterology 142: 957-966 $\mathrm{e} 12,2012$.

12. Robinson MD, McCarthy DJ and Smyth GK: edgeR: A Bioconductor package for differential expression analysis of digital gene expression data. Bioinformatics 26: 139-140, 2010.

13. Gene Ontology Consortium: The Gene Ontology (GO) project in 2006. Nucleic Acids Res 34 (Database Issue): D322-D326, 2006.

14. Kanehisa M and Goto S: KEGG: Kyoto encyclopedia of genes and genomes. Nucleic Acids Res 28: 27-30, 2000.

15. Huang da W, Sherman BT and Lempicki RA: Systematic and integrative analysis of large gene lists using DAVID bioinformatics resources. Nat Protoc 4: 44-57, 2009.

16. Szklarczyk D, Franceschini A, Wyder S, Forslund K, Heller D, Huerta-Cepas J, Simonovic M, Roth A, Santos A, Tsafou KP, et al: STRING v10: Protein-protein interaction networks, integrated over the tree of life. Nucleic Acids Res 43 (Database Issue) D447-D452, 2015.

17. Gentleman RC, Carey VJ, Bates DM, Bolstad B, Dettling M, Dudoit S, Ellis B, Gautier L, Ge Y, Gentry J, et al: Bioconductor: Open software development for computational biology and bioinformatics. Genome Biol 5: R80, 2004

18. Hong G, Zhang W, Li H, Shen X and Guo Z: Separate enrichment analysis of pathways for up- and downregulated genes. J R Soc Interface 11: 20130950, 2013

19. Chen P, Zheng X, Zhou Y, Xu Y, Zhu L and Qian Y: Talin-1 interaction network promotes hepatocellular carcinoma progression. Oncotarget 8: 13003-13014, 2017.

20. Sun H, Peng Z, Tang H, Xie D, Jia Z, Zhong L, Zhao S, Ma Z Gao Y, Zeng L, et al: Loss of KLF4 and consequential downregulation of Smad7 exacerbate oncogenic TGF- $\beta$ signaling in and promote progression of hepatocellular carcinoma. Oncogene 36: $2957-2968,2017$

21. Ko JH, Ko EA, Gu W, Lim I, Bang H and Zhou T: Expression profiling of ion channel genes predicts clinical outcome in breast cancer. Mol Cancer 12: 106, 2013

22. Kulkarni A, Natarajan SK, Chandrasekar V, Pandey PR and Sengupta S: Combining immune checkpoint inhibitors and kinase-inhibiting supramolecular therapeutics for enhanced anticancer efficacy. Acs Nano, 2016.

23. Hughes PE, Caenepeel S and Wu LC: Targeted therapy and checkpoint immunotherapy combinations for the treatment of cancer. Trends Immunol 37: 462-476, 2016.

24. Hanahan D and Weinberg RA: The hallmarks of cancer. Cell 100: $57-70,2000$

25. Hanahan D and Weinberg RA: Hallmarks of cancer: The next generation. Cell 144: 646-674, 2011.
26. Dominguez-Brauer C, Thu KL, Mason JM, Blaser H, Bray MR and Mak TW: Targeting mitosis in cancer: Emerging strategies. Mol Cell 60: 524-536, 2015

27. Visconti R, Della Monica R and Grieco D: Cell cycle checkpoint in cancer: A therapeutically targetable double-edged sword. J Exp Clin Cancer Res 35: 153, 2016.

28. Cai H, Li H, Li J, Li X, Li Y, Shi Y and Wang D: Sonic hedgehog signaling pathway mediates development of hepatocellular carcinoma. Tumour Biol, 2016.

29. Jin B, Wang W, Du G, Huang GZ, Han LT, Tang ZY, Fan DG, Li J and Zhang SZ: Identifying hub genes and dysregulated pathways in hepatocellular carcinoma. Eur Rev Med Pharmacol Sci 19: 592-601, 2015

30. Bertoli C, Skotheim JM and de Bruin RA: Control of cell cycle transcription during G1 and S phases. Nat Rev Mol Cell Biol 14: 518-528, 2013.

31. Fang Y, Yu H, Liang X, Xu J and Cai X: Chk1-induced CCNB1 overexpression promotes cell proliferation and tumor growth in human colorectal cancer. Cancer Biol Ther 15: 1268-1279, 2014.

32. Ding K, Li W, Zou Z, Zou X and Wang C: CCNB1 is a prognostic biomarker for ER+ breast cancer. Med Hypotheses 83: 359-364, 2014.

33. Diab A, Foca A, Fusil F, Lahlali T, Jalaguier P, Amirache F, N'Guyen L, Isorce N, Cosset FL, Zoulim F, et al: Polo-like-kinase 1 is a proviral host factor for hepatitis B virus replication. Hepatology 66: 1750-1765, 2017.

34. Li J, Wang R, Gannon OJ, Rezey AC, Jiang S, Gerlach BD, Liao G and Tang DD: Polo-like Kinase 1 regulates vimentin phosphorylation at ser-56 and contraction in smooth muscle. J Biol Chem 291: 23693-23703, 2016.

35. Ruf S, Heberle AM, Langelaar-Makkinje M, Gelino S, Wilkinson D, Gerbeth C, Schwarz JJ, Holzwarth B, Warscheid B, Meisinger C, et al: PLK1 (polo like kinase 1) inhibits MTOR complex 1 and promotes autophagy. Autophagy 13: 486-505, 2017.

36. Slamon DJ and Press MF: Alterations in the TOP2A and HER2 genes: Association with adjuvant anthracycline sensitivity in human breast cancers. J Natl Cancer Inst 101: 615-618, 2009.

37. Brase JC, Schmidt M, Fischbach T, Sültmann H, Bojar H, Koelbl H, Hellwig B, Rahnenführer J, Hengstler JG and Gehrmann MC: ERBB2 and TOP2A in breast cancer: A comprehensive analysis of gene amplification, RNA levels, and protein expression and their influence on prognosis and prediction. Clin Cancer Res 16: 2391-2401, 2010

38. Garces de Los Fayos Alonso I, Liang HC, Turner SD, Lagger S, Merkel $\mathrm{O}$ and Kenner L: The role of activator protein-1 (AP-1) family members in cd30-positive lymphomas. Cancers (Basel) 10: pii: E93, 2018

39. Carmena M and Earnshaw WC: The cellular geography of aurora kinases. Nat Rev Mol Cell Biol 4: 842-854, 2003

40. Kapanidou M, Curtis NL and Bolanos-Garcia VM: Cdc20: At the crossroads between chromosome segregation and mitotic exit. Trends Biochem Sci 42: 193-205, 2017.

41. Hingorani P, Dickman P, Garcia-Filion P, White-Collins A, Kolb EA and Azorsa DO: BIRC5 expression is a poor prognostic marker in Ewing sarcoma. Pediatr Blood Cancer 60: 35-40, 2013.

42. Wang Q, Shu R, He H, Wang L, Ma Y, Zhu H, Wang Z, Wang S, Shen G and Lei P: Co-silencing of Birc5 (survivin) and Hspa5 (Grp78) induces apoptosis in hepatoma cells more efficiently than single gene interference. Int J Oncol 41: 652-660, 2012.

43. Yang W, Cho H, Shin HY, Chung JY, Kang ES, Lee EJ and Kim JH: Accumulation of cytoplasmic Cdk1 is associated with cancer growth and survival rate in epithelial ovarian cancer. Oncotarget 7: 49481-49497, 2016.

44. Willder JM, Heng SJ, McCall P, Adams CE, Tannahill C, Fyffe G, Seywright M, Horgan PG, Leung HY, Underwood MA and Edwards J: Androgen receptor phosphorylation at serine 515 by Cdk1 predicts biochemical relapse in prostate cancer patients. Br J Cancer 108: 139-148, 2013.

45. Sung WW, Lin YM, Wu PR, Yen HH, Lai HW, Su TC, Huang RH, Wen CK, Chen CY, Chen CJ and Yeh KT: High nuclear/cytoplasmic ratio of $\mathrm{Cdk} 1$ expression predicts poor prognosis in colorectal cancer patients. BMC Cancer 14: 951, 2014. International (CC BY-NC-ND 4.0) License. 